\title{
ALTENBURG, Tilo, Soziale Ordnungsvorstellungen bei Hildegard von Bingen
}

\section{Laurence Moulinier-Brogi}

\section{OpenEdition}

\section{Journals}

Édition électronique

URL : http://journals.openedition.org/ifha/1793

DOI : 10.4000/ifha.1793

ISSN : 2198-8943

\section{Éditeur}

IFRA - Institut franco-allemand (sciences historiques et sociales)

\section{Référence électronique}

Laurence Moulinier-Brogi, «ALTENBURG, Tilo, Soziale Ordnungsvorstellungen bei Hildegard von Bingen », Revue de l'IFHA [En ligne], Date de recension, mis en ligne le 01 janvier 2009, consulté le 22 septembre 2020. URL : http://journals.openedition.org/ifha/1793 ; DOI : https://doi.org/10.4000/ifha.1793

Ce document a été généré automatiquement le 22 septembre 2020

(CIFHA 


\title{
ALTENBURG, Tilo, Soziale Ordnungsvorstellungen bei Hildegard von Bingen
}

\author{
Laurence Moulinier-Brogi
}

L'œuvre abondante et diverse de Hildegarde de Bingen (1098-1179) est étudiée au prisme de problématiques de plus en plus variées, voire raffinées, mais la question des idées que la visionnaire avait de la société n'avait jamais fait l'objet d'une synthèse. Le livre de T.A. vient donc combler un manque, l'auteur s'étant donné pour objectif d'examiner, dans l'ensemble des écrits de Hildegarde, la conception qu'elle avait de différents groupes ou, mieux, de différents états de la société. Pour ce faire, il part des représentations de la société qui avaient alors cours : la majorité des schémas en vigueur à l'époque reposaient sur une dualité ou sur une tripartition, et un chapitre introductif s'emploie à les passer en revue. La dichotomie entre spirituales et seculares, accentuée depuis la réforme grégorienne, parcourt ainsi l'œuvre de Hildegarde, en se combinant ou en s'opposant à un autre clivage, à savoir le schéma tripartite répartissant les êtres humains selon leur degré de continence : vierges, veuves et mariés. Mais les spirituales à leur tour se divisent, par exemple entre moines et chanoines, en compétition accrue depuis l'apparition d'expériences spirituelles innovantes et diversifiées comme les cisterciens ou les prémontrés, ou entre moines et nonnes ; quant aux seculares, ils se scindent entre puissants et faibles, pauvres et riches, stulti et sapientes, nobles et non-nobles - cette dernière dichotomie étant la plus problématique, l'apparition des ministeriales, propre à l'Allemagne, venant compliquer par exemple l'opposition reçue entre liberi et servi.

La recherche de T.A. suit un ordre chronologique et le livre se décompose en trois parties principales (chap. II-IV) correspondant aux trois périodes de la créativité littéraire de Hildegarde que permettent de distinguer ses trois grands écrits visionnaires, Scivias, Liber vite meritorum et Liber divinorum operum. Des souschapitres de moindre longueur plantent en quelque sorte le décor ou explicitent le Menschenbild de Hildegarde. Chaque chapitre se conclut par un résumé, et le chapitre $\mathrm{V}$ est à son tour une sorte de gros résumé des acquis de ce travail très structuré, dans 
lequel des index achèvent de permettre de s'orienter facilement. Hildegarde a écrit de fait pendant plus de trente ans, de 1141 à sa mort en 1179, et le découpage de T.A. se justifie par sa volonté d'observer l'évolution d'une pensée : pour n'en donner qu'un bref aperçu, l'opposition entre moines et chanoines réguliers, sensible dans le Scivias terminé en 1151, s'atténue dans les écrits ultérieurs, comme si cette querelle avait été réglée ou apaisée, ce qui est pleinement manifeste dans la dernière œuvre visionnaire, le Liber divinorum operum achevé en 1173. Comme il s'en explique, son travail n'est pas fondé sur des recherches en archives, mais sur la scrutation attentive d'une pensée dans le temps - au détriment, peut-être, de comparaisons plus poussées avec des œuvres contemporaines ou comparables, ainsi l'Hortus deliciarum de Herrade, qui ne fait l'objet que de deux notes, ou le Speculum virginum (p. 103). T.A. n'exclut pas tout renvoi à d'autres pensées, telle celle de Rupert de Deutz, mais la particularité du Hildegard-Problem l'amène à se concentrer sur l'œuvre de la magistra du Rupertsberg, ce qui nous vaut par exemple un chapitre très fouillé sur la Vie de son saint patron écrite par Hildegarde, la Vita sancti Ruperti et ejus matris Berthae (p. 327-340), de fines remarques sur l'hétérogénéité de sa correspondance en tant que corpus, ou encore des pages éclairantes sur les tournées de prédication entreprises par la nonne dans les années 1163-1165 et les problèmes méthodologiques afférents.

Faute de pouvoir rendre compte de la richesse des thèmes abordés, on en privilégiera deux, qui s'imposent comme des fils rouges, avec les variations qu'ils purent connaître dans l'esprit de Hildegarde en plus de trois décennies : la noblesse et la virginité.

Elle-même d'origine noble, Hildegarde faisait un lien entre noblesse de la naissance et vertu, et avait une haute conscience de sa noblesse. C'est ce que montre entre autres sa lettre à Tenxwind d'Andernach (vers 1148-1150) qui a déjà fait couler beaucoup d'encre et est devenue en quelque sorte le paradigme de cet Adelbewußtsein (Hildegardis Bingensis, Epistolarium. Pars prima, éd. L. van Acker, Turnhout, 1991, Ep. 52R, p. 40 45, trad. L. Moulinier, "Hildegarde de Bingen, Chants et Lettres (choix)» in : D. Régnier-Bohler (dir.) Voix de femmes au Moyen Age. Savoir, mystique, poésie, amour, sorcellerie XIIe-XVe siècle, Paris, 2006, p. 77-124, p. 119). Cette magistra reprochait à Hildegarde de ne pas accepter dans sa communauté de jeunes filles d'origine non noble, et celle-ci se justifia notamment par une formule frappante : «Dieu en effet examine et scrute minutieusement chaque personne, de sorte que l'ordre inférieur ne s'élève pas au-dessus de l'ordre supérieur, comme le firent Satan et le premier homme, en voulant voler plus haut qu'ils n'avaient été placés. Et quel homme rassemblerait tout son troupeau dans une même étable, à savoir, les bœufs, les ânes, les brebis et les boucs, sans courir le risque qu'ils ne se battent? ?. Et elle poursuit : « il faut éviter surtout que la décence morale ne soit mise à mal et que la haine ne le déchire, lorsque les plus nobles tombent plus bas que les plus humbles, et que ces derniers s'élèvent au-dessus du rang qui leur est supérieur ». La magistra du Rupertsberg revendiquait en effet une inégalité entre les êtres voulue par Dieu (« Et Dieu les aime tous, mais ils n'en ont pas pour autant des noms égaux »); face à elle, Tenxwind représentait une mentalité plus neuve, dans l'esprit des mouvements de pauvreté qui s'étaient fait jour depuis le XIe s. : issue d'une famille de ministeriales, elle avait en outre pour frère le chef de la communauté de chanoines réguliers de Springiersbach. C'est l'occasion pour T.A. de faire une brève revue bibliographique autour de cet échange épistolaire, et de se situer dans un débat réactivé par les récentes célébrations autour du 900ème anniversaire de la nonne. Alfred Haverkamp avait caractérisé les deux femmes comme représentatives de deux Weltanschauungen opposées, le nouvel esprit religieux contre le vieux 
monachisme bénédictin (A. Haverkamp, « Tenxwind von Andernach und Hildegard von Bingen. Zwei „Weltanschauungen“ in der Mitte des zwölften Jahrhunderts » in : L. Fenske, W. Rösener, T. Zotz (dir.), Institutionen, Kultur und Gesellschaft im Mittelalter. Festschrift für Josef Fleckenstein zu seinem 65. Geburtstag, Sigmaringen, 1984, p. 515548). Mais Franz Staab, de son côté, a rejeté cette interprétation comme une lecture strictement philologique de l'échange, et a attiré l'attention sur les relations de Hildegarde avec les lignées de ministériaux des Bolander et des Embricho, pour émettre l'hypothèse qu'il y avait aussi des filles d'origine non libre dans le couvent de Hildegarde (F. Staab, " Geringschätzung der Ministerialen? Die Beziehung der Rheingrafen und der Bolander zu Hildegard von Bingen und zum Rupertsberg bis zum Beginn des 13. Jahrhunderts » in : W. Podehl (dir.), 900 Jahre Hildegard von Bingen. Neuere Untersuchungen und literarische Nachweise, Wiesbaden, 1998, p. 45-65, p. 58). Sabina Flanagan lui a emboîté le pas, mais la critique n'a pourtant pas totalement convaincu, dans la mesure où la " conscience de la noblesse » de Hildegarde se trouve ailleurs, par exemple dans son Scivias ou dans les passages autobiographiques de sa Vita (S. Flanagan, « For God distinguishes the People of Earth as in Heaven : Hildegard of Bingen's Social Ideas ", Journal of Religious History, 22, 1998, p. 14-34). Enfin, comme le souligne Altenburg, s'il est vrai que les Bolander étaient des ministériaux, ils étaient d'origine noble, ce qu'on n'a peut-être pas assez pris en considération pour apprécier les rapports de Hildegarde avec ce groupe auquel il consacre des pages détaillées et informées (p. 253-264).

La fameuse lettre à Tenxwind montre aussi que Hildegarde avait développé, dans la vie spirituelle de sa communauté, des pratiques très éloignées de celles que lui avait inculquées sa première maîtresse, la recluse Jutta de Spanheim, par exemple en ce qui concerne la parure des nonnes. Très loin des idées ascétiques de Jutta, les moniales du Rupertsberg étaient vêtues d'un vêtement blanc, comme toutes les vierges dans les écrits visionnaires de Hildegarde, et portaient un voile, autant dire une traîne allant jusqu'aux pieds (p. 102, 201) : «C'est pourquoi il est juste, comme l'a révélé et autorisé le souffle mystique du doigt de Dieu, qu'une vierge porte des habits blancs, symbole éclatant de ses fiançailles avec le Christ ", écrit-elle encore à Tenxwind pour se justifier. Et c'est l'occasion pour T.A. de souligner avec justesse combien Hildegarde avait pris ses distances avec sa première formatrice que, significativement, elle ne nomme jamais virgo (p. 109).

Un des atouts du livre de T.A. est que, outre qu'il passe véritablement au crible une œuvre, à la recherche de lignes de force et de nuances, il sait également la mettre finement en relation avec l'histoire locale et avec son contexte historique immédiat en s'appuyant par exemple sur une source éditée d'importance comme le Mainzer Urkundenbuch. On renverra le lecteur à son analyse de l'impact de la fondation du Rupertsberg en 1150, donc de la sécession d'avec le Disibodenberg, sur la conception déjà très élevée que Hildegarde se faisait de la virginité au féminin, ou à son étude des liens de Hildegarde avec les adversaires de l'archevêque de Mayence Arnold (p. 272-287).

Partant du présupposé que la position de Hildegarde envers les non-libres aurait évolué, l'auteur, on l'a dit, analyse de très près l'histoire des familles ministérielles des comtes palatins et des Bolander. Nombre de positions affirmées dans le Scivias, lors de la " première période " de Hildegarde, pour ainsi dire, apparaissent dépassées ou enterrées dans les œuvres qui suivent, et T.A. suggère que les dons reçus par le Rupertsberg de la part de bourgeois ou de ministériaux, ou encore le départ de 
Richardis von Stade, qui venait d'être nommée abbesse de Bassum, ont pu influencer sa façon de voir la noblesse. On ne résiste pas à cette occasion au plaisir de donner la parole à Hildegarde d'après la lettre qu'elle adresse à Richarde en 1151-1152 :

"Écoute-moi, ô ma fille, moi ta mère, qui te dis dans l'Esprit saint : " Ma douleur s'élève. La douleur a tué la grande confiance que j'avais placée en un être et le réconfort que j'y trouvais ». Dorénavant, je dirai : «Il est bon de placer son espoir dans le Seigneur plutôt que dans les princes ». Ce qui signifie : Chacun doit tourner ses regards vers la vivante hauteur sans souffrir de l'ombre portée par un amour et une confiance de peu de force, liées à l'humeur aérienne de la terre, et qui ne sauraient durer. On doit regarder Dieu comme l'aigle pose son regard sur le soleil. Et pour cette raison, on ne doit pas placer ses désirs dans une personne de haut rang qui vient à manquer comme une fleur qui tombe. Et j'ai transgressé ce précepte pour l'amour d'une noble personne " (Hildegardis Bingensis, Epistolarium. Pars prima, Ep. 64, pp. 147-148, trad. L. Moulinier, « Hildegarde de Bingen, Chants et Lettres (choix)», p. 111).

T.A. remarque aussi, en se fondant sur une analyse fine du vocabulaire, qu'après le véritable drame qu'a représenté pour Hildegarde le départ de sa moniale préférée, elle ne qualifie plus le Christ de nobilis dans ses écrits (p. 373).

En ce qui concerne nonnes et moines, T.A. reconnaît qu'il y a une césure au sein même du Scivias : si Hildegarde les considère tout du long comme un groupe unifié, elle les sépare dans son troisième livre, et ce sont les virgines de sexe féminin qui obtiennent la place la plus élevée dans sa représentation de l'humanité. Il est clair que Hildegarde créditait les femmes d'une plus grande capacité à conserver leur pudeur, et que leurs dispositions physiques aussi jouaient dans ce sens. Aux yeux de T.A., ce changement de cap et cette suprématie clairement reconnue au Dasein virginal des femmes pourrait précisément être lié à la rupture d'avec le Disibodenberg. Tant qu'elle écrit au Disibodenberg, moines et nonnes sont présentés sous sa plume comme un même groupe (et l'on ne saurait oublier l'importance à l'époque des monastères doubles dans ces terres d'Empire), mais ce n'est plus le cas une fois qu'elle a fondé sa propre communauté féminine. En tant que membres du sexe faible, les femmes, à ses yeux, n'atteignent pas la perfection en devenant hommes, en se faisant viragines comme c'était jusqu'alors le cas, mais bel et bien par leur féminité même (p. 108 suiv.). Hildegarde a pourtant bien des traits traditionnels dans la représentation qu'elle se fait de la femme en général, qui doit, selon elle, obéir à son mari comme un valet à son seigneur ; mais cela ne vaut pas pour les religieuses. "Ainsi la femme ne doit pas se mettre en valeur et s'arranger en apprêtant ses cheveux, ni chercher à se rehausser en portant des diadèmes ou des bijoux en or, à moins que ce ne soit la volonté de son homme, et elle doit alors s'y plier pour lui plaire dans une juste mesure. Cela n'est toutefois pas valable pour les vierges : elles se tiennent dans la simplicité et l'intégrité de la beauté du paradis, qui jamais n'apparaîtra comme aride, mais demeure toujours dans la plénitude de la verdeur de la fleur de la branche. La vierge ne couvre pas ses cheveux, symbole de sa verdeur, en vertu d'un commandement, mais elle se couvre de son propre chef, en vertu de sa suprême humilité, de même que tout un chacun cachera la beauté de son âme, de peur que l'oiseau de proie ne s'en empare par orgueil ", assène-t-elle encore à Tenxwind.

Enfin, comme le relève également avec pénétration l'auteur (p. 207), l'une des nouveautés du Rupertsberg par rapport au Disibodenberg est la présence d'un groupe 
de veuves, qui viennent grossir les rangs de la perfection spirituelle au féminin et à qui Hildegarde dédiera notamment un de ses chants, la Symphonia viduarum.

On l'aura compris, T.A. offre à la réflexion le produit d'une lecture remarquablement fouillée et attentive de l'œuvre profuse de Hildegarde, et des analyses très fines, certes parfois un peu dispersées, au risque du pointillisme. À propos de l'ascèse que refuse Hildegarde, par exemple, les commentaires auraient gagné à être regroupés, et l'auteur aurait pu rappeler que ce thème parcourt tant la direction de conscience que Hildegarde exerça auprès d'Elisabeth de Schönau que l'action qu'elle mena contre les cathares. La lecture de l'ouvrage d'Uwe Brunn aurait pu nourrir cette réflexion mais il est vrai que la recherche non anglo- ou germanophone est étonnamment absente de la bibliographie (U. Brunn, Des contestataires aux cathares, discours de réforme et propagande antihérétique dans les pays du Rhin et de la Meuse avant l'Inquisition, Paris, 2006, chap. V). Ce qui n'enlève rien au principal mérite de ce travail, qui est d'avoir réussi à se frayer une voie innovante dans le champ désormais très labouré de la recherche hildegardienne. Incontestablement, les pages de ce livre ouvrent des perspectives stimulantes, voire les clés d'une compréhension à nouveaux frais de la vie et de l'œuvre hors norme de la visionnaire.

Laurence MOULINIER-BROGI (Université Lumière Lyon II) 